\title{
Activités analgésique, antiinflammatoire et antipyrétique d'un extrait aqueux des tourteaux de la pomme de cajou (Anacardium occidentale $\mathbf{L}$.)
}

\author{
Mamadou DOSSO ${ }^{1 *}$, Allali Eugène KOFFI ${ }^{2}$, Doudjo $\mathrm{SORO}^{3,}$ Ali TRAORE ${ }^{1}$ et \\ Nafan DIARRASSOUBA ${ }^{1}$
}

\begin{abstract}
${ }^{1}$ Unité de Formation et de Recherche des Sciences Biologiques, Département Biochimie-Génétique, Université Peleforo GON COULIBALY de Côte d'Ivoire, BP 1328, Korhogo-CI, Côte d'Tvoire.

${ }^{2}$ UFR Agroforesterie, Laboratoire d'Agrovalorisation, Département de Biochimie et de Microbiologie,

Université Jean Lorougnon GUEDE, BP 150, Daloa, Côte d'Ivoire.

${ }^{3}$ UMRI Sciences des Procédés Alimentaires, Chimiques et Environnementaux, Institut National Polytechnique Félix Houphouët-Boigny (INP-HB), BP 1313, Yamoussoukro, Côte d'Ivoire.

*Auteur correspondant ; E-mail : coatcha1012@gmail.com
\end{abstract}

\begin{tabular}{ccc}
\hline Received: 05-06-2021 & Accepted: 07-10-2021 & Published: 30-10-2021 \\
\hline
\end{tabular}

\section{RESUME}

Les anti-inflammatoires non stéroïdiens (AINS), bien qu'efficients, provoquent des ulcères, des insuffisances rénales, des bronchospasmes et des hépato-toxicités chez certains patients. Dans le cadre de la recherche d'une alternative à l'utilisation de ces molécules de synthèses, les agro-ressources notamment la pomme de cajou, contenant des molécules bioactives d'intérêts pharmacologiques, pourrait être d'une utilité réelle. Ainsi, il s'est agi au cours de ce travail, d'évaluer les effets de l'extrait aqueux des tourteaux de la pomme de cajou (CAJ) sur la douleur, l'inflammation et la fièvre. L'activité analgésique a été évaluée sur la douleur induite par injection de l'acide acétique chez la souris. L'activité anti-inflammatoire a été quant à elle, évaluée sur un modèle d'œdème induit par injection de la carragénine sous l'aponévrose plantaire de la patte postérieure droite du rat. S'agissant de l'activité antipyrétique, elle a été évaluée chez la souris rendue hyperthermique par la levure de bière injectée dans la zone dorsolatérale. CAJ pour des concentrations comprises entre $75 \mathrm{mg} / \mathrm{Kg}$ PC et $150 \mathrm{mg} / \mathrm{Kg} \mathrm{PC}$, diminue significativement les contorsions induites par l'acide acétique, chez la souris. En présence de la naloxone, cet effet protecteur de CAJ, diminue de 6,9\% à 7,07\%, valeurs non significatives. Au bout de quatre heures, $\mathrm{CAJ}$, réduit l'hyperthermie provoquée par la levure de bière chez la souris. CAJ pour des concentrations allant de $100 \mathrm{mg} / \mathrm{Kg}$ PC à $300 \mathrm{mg} / \mathrm{Kg}$ PC, diminue l'augmentation de l'œdème de la patte du rat. Ces résultats montrent que CAJ possède des activités analgésiques périphériques, antipyrétiques et antiinflammatoires. Il pourrait être, de ce fait un atout en médecine traditionnelle.

(C) 2021 International Formulae Group. All rights reserved.

Mots clés : Analgésique, antipyrétique, antiinflammatoire, pomme de cajou, souris, rat.

\section{Analgesic, anti-inflammatory and antipyretic activities of an aqueous extract of cashew apple cake (Anacardium occidentale L.)}

\begin{abstract}
Non-steroidal anti-inflammatory drugs (NSAIDs), although effective, cause ulcers, renal failure, bronchospasm and hepatotoxicity in some patients. In the context of the search for an alternative to the use of
\end{abstract}


these synthetic molecules, agro-resources, in particular cashew apples, containing bioactive molecules of pharmacological interest, could be of real use. Thus, the aim of this work was to evaluate the effects of the aqueous extract of this apple cake (CAJ) on pain, inflammation and fever. The analgesic activity was evaluated on pain induced by acetic acid injection in mice. The anti-inflammatory activity was evaluated on a model of edema induced by injection of carrageenan under the plantar fascia of the right hind leg of rats. The antipyretic activity was evaluated in mice made hyperthermic by brewer's yeast injected in the dorsolateral zone. CAJ for concentrations between $75 \mathrm{mg} / \mathrm{Kg} \mathrm{BW}$ and $150 \mathrm{mg} / \mathrm{Kg} \mathrm{BW}$, significantly reduces acetic acid-induced contortions in mice. In the presence of naloxone, this protective effect of CAJ, decreases from $6.9 \%$ to $7.07 \%$, non-significant values. After four hours, CAJ, reduces brewer's yeast-induced hyperthermia in mice. CAJ for concentrations ranging from $100 \mathrm{mg} / \mathrm{Kg} \mathrm{BW}$ to $300 \mathrm{mg} / \mathrm{Kg} \mathrm{BW}$, decreases the increase in edema of the rat paw. These results show that CAJ has peripheral analgesic, antipyretic and anti-inflammatory activities. Therefore, it could be an asset in traditional medicine.

(C) 2021 International Formulae Group. All rights reserved.

Keywords: Analgesic, antipyretic, anti-inflammatory, cashew apple, mouse, rat.

\section{INTRODUCTION}

L'efficacité des anti-inflammatoires non stéroïdiens (AINS) à soulager la fièvre, les douleurs et les inflammations, explique le plus grand nombre de leur prescription. Toutefois, il est récurrent qu'ils provoquent des effets indésirables qui suscitent de réelles préoccupations. Les salicylés, antiinflammatoires antipyrétiques inhibant les COX1 en plus des COX2, provoquent des ulcères, des insuffisances rénales et des bronchospasmes chez des asthmatiques; l'acétaminophène, antalgique antipyrétique non salicylé, dérivé de la phénacétine, retiré du marché, expose l'individu à des hépatotoxicités, au-delà des doses recommandées ; les coxibs (rofécoxib et célécoxib), AINS de deuxième génération, qui ont l'avantage d'inhiber de manière sélective les COX2, responsables de la synthèse des prostaglandines «physiopathologiques », augmentent les risques d'accidents cardiaques (Corrado et al., 2009 ; Ramalawi et al., 2013). Fort de ce constat, il apparait opportun de trouver des voies alternatives, d'où l'intérêt porté aux agro-ressources qui seraient dotés d'effets thérapeutiques indéniables y compris contre les douleurs, la fièvre et les réactions inflammatoires, avec moins d'effets secondaires. Le raisin est un régulateur des troubles d'hypertension artérielle. La pomme traite les inflammations de l'estomac et de l'intestin, ainsi que les affections du foie (Zouaouin, 2005; Chira et al., 2008). Le contexte indiqué, explique le choix de la pomme de cajou, faux fruit d'Anacardium occiental L. Arbre de 8 à 10 mètres de haut, l'Aanacardium Occidentale L. appartient à la famille des anacardiacées dont font également partie le manguier (Mangifera indica L.) et le pistachier (Pistacia vera L.). Avec un diamètre pouvant atteindre 12 à 15 mètres, il possède un feuillage dense et ramifié. Le fruit, la noix de cajou, pend au bout d'un pédoncule charnu, juteux, de couleur rouge ou jaune à maturité appelé pomme de cajou (Soro, 2012).

En médecine traditionnelle, l'huile caustique des noix traite les dartres. Le décocté de l'écorce est utilisé comme un antihypertenseur et un anti-dysentérique. Le fruit soulage les ulcères et les maux de dents (Tédong, 2007). La pomme est un agent antigrippal et antidiabétique.

Selon des études pharmacologiques, l'extrait aqueux de la pomme de cajou, doué d'activité glycémique autorégulatrice, contient des saponosides et des flavonoïdes (Dosso et al., 2021).

Pour donc contribuer à enrichir ces données pharmacologiques, il s'agissait dans cette étude d'évaluer les activités analgésique, anti-inflammatoire et antipyrétique de l'extrait aqueux de la pomme de cajou. 


\section{MATÉRIEL ET MÉTHODES}

\section{Matériel}

\section{Matériel végétal}

Dans le courant du mois de décembre 2019, les pommes de cajou ont été récoltées sur les arbres ou ramassées après leur chute. Provenant de Korhogo, une ville située à l'extrême nord de la Cote d'Ivoire, les pommes de couleur rouge ou jaune sans aucune blessure, ont été sélectionnées. Ensuite, elles ont été séparées minutieusement des noix, puis transportées dans des paniers au laboratoire.

\section{Matériel animal}

Des souris de l'espèce Mus musculus mâles et femelles de poids compris entre 20 et $30 \mathrm{~g}$ ont été utilisées pour les tests toxicologiques, analgésiques et antipyrétiques. Elles proviennent de l'animalerie de l'institut Pasteur d'Adiopodoumé situé sur la route de Dabou (Côte d'Ivoire). Transportées à l'animalerie de l'université Peleforo GON COULIBALY, elles y ont été élevées à une température moyenne de $28{ }^{\circ} \mathrm{C}$ avec une humidité relative de $70 \%$ et nourries aux granulés.

Des rats albinos de souche Wistar ont été utilisés pour des tests anti-inflammatoires. Ils pèsent entre 200 et $300 \mathrm{~g}$. Provenant également de l'institut Pasteur d'Adiopodoumé, ils ont été élevés à l'animalerie de l'Université Peleforo GON COULIBALY.

\section{Produits chimiques et substances naturelles}

Acétylsalicylate de lysine (ASPEGIC): Sanofi-SyntheLabo (France). Diclofénac Sodium 75mg/3ml Denk Pharma (Germany). Naloxone Aguettant $0,4 \mathrm{mg} / \mathrm{mL}$, injectable. Acide acétique: Merck (Allemagne). Levure de bière: ArkoPharma Belux s.a. (France). Carragénine (Carrageenan): Wako pure chemical Industrie, Ltd (Japon).

\section{Méthodes}

\section{Préparation de l'extrait aqueux}

Les pommes recueillies sont nettoyées, lavées puis désinfectées pendant $30 \mathrm{~min}$ au chlore actif dans des bacs. Ensuite, elles sont rincées avec de l'eau avant d'être pressées. Le pressage et l'essorage sont réalisés manuellement. Les tourteaux séparés du jus, ont été séchés à l'ombre entre 25 et $28{ }^{\circ} \mathrm{C}$. Séchés, ces tourteaux sont broyés et réduits en poudre. Cette poudre a servi à la préparation de l'extrait aqueux de pomme de cajou. Cinquante grammes $(50 \mathrm{~g})$ de broyat de pomme de cajou sont mixées sur un agitateur magnétique de type AGIMATIC-N, pendant 24 heures dans un litre d'eau distillée. La solution obtenue est filtrée à l'aide du coton hydrophile et sur papier Wattman. De l'eau distillée est ajoutée au culot, puis mixée pendant 2 heures et filtrée également. Les filtrats sont recueillis dans un ballon et séchés à l'étuve à $60{ }^{\circ} \mathrm{C}$. La poudre obtenue, parfaitement soluble dans l'eau, est utilisée comme l'extrait aqueux de pomme de cajou (CAJ).

\section{Toxicité aigüe par injection intrapéritonéale}

Les souris ont été placées dans des cages quelques jours avant l'étude de la toxicité aigüe de CAJ. Cette étude de toxicité a nécessité l'utilisation de 5 lots de 10 souris vigiles de poids corporel compris entre 18 et 30 grammes. Chaque lot contenait autant de mâles que de femelles. CAJ a été dilué dans des solutions isotoniques de $\mathrm{NaCl}$ à $0,9 \%$. Quatre lots de souris ont reçu par injection intra péritonéale, des doses uniques croissantes de CAJ, comprises entre 100 et $10^{3} \mathrm{mg} / \mathrm{Kg}$ PC. Le lot témoin n'a reçu que du Nacl isotonique à $0,9 \%$.

Après administration de ces différentes solutions, les souris ont été observées pendant deux heures, puis alimentées. Ensuite, Elles sont observées pendant 24 heures puis sur deux semaines. Pendant cette période d'observation, le comportement général des souris en cage, les modifications du rythme respiratoire et le nombre de mort, constituent les paramètres observés.

\section{Activité analgésique de CAJ}

Cette étude a été réalisée selon la méthode du test de writhing, utilisée par Soro et al. (2009). Il s'est agi de compter le nombre de contorsions abdominales induites par l'injection de l'acide acétique $(0,6 \%)$, chez la 
souris, en présence de la substance test, l'eau distillée ou un analgésique de référence.

Cinq (5) lots de souris, selon que chaque lot contenant 5 animaux, ont été constitués :

$\checkmark$ Lot 1: souris traitées avec de l'eau distillée, dix minutes avant l'injection de l'acide acétique $(0,6 \%)$ par voie intrapéritonéale.

$\checkmark \quad$ Lot 2: souris traitées avec du tramadol à $15 \mathrm{mg} / \mathrm{Kg} \mathrm{PC}$, dix minutes avant l'injection de l'acide acétique par voie intrapéritonéale.

$\checkmark \quad$ Lot 3: souris traitées avec de l'aspirine $75 \mathrm{mg} / \mathrm{Kg} \mathrm{PC}$, dix minutes avant l'injection de l'acide acétique par voie intrapéritonéale.

$\checkmark \quad$ Lot 4: souris traitées avec du CAJ à 75 $\mathrm{mg} / \mathrm{Kg} \quad \mathrm{PC}$, dix minutes avant l'injection de l'acide acétique par voie intrapéritonéale.

$\checkmark \quad$ Lot 5: souris traitées avec du CAJ à $150 \mathrm{mg} / \mathrm{Kg}$ PC, dix minutes avant l'injection de l'acide acétique par voie intrapéritonéale.

\section{Activité analgésique de CAJ en présence de la noloxone}

Cinq (5) lots de souris, selon que chaque lot contenant 5 animaux, ont été constitués :

$\checkmark$ Lot 1: souris traitées avec de la naloxone et l'eau distillée, dix minutes avant l'injection de l'acide acétique $(0,6 \%)$ par voie intrapéritonéale.

$\checkmark$ Lot 2: souris traitées avec de la naloxone et du tramadol à $15 \mathrm{mg} / \mathrm{Kg}$ PC, dix minutes avant l'injection de l'acide acétique par voie intrapéritonéale.

$\checkmark$ Lot 3: souris traitées avec de la naloxone et l'aspirine à $75 \mathrm{mg} / \mathrm{Kg} \mathrm{PC}$, dix minutes avant l'injection de l'acide acétique par voie intrapéritonéale.

$\checkmark$ Lot 4: souris traitées avec de la naloxone et CAJ à $75 \mathrm{mg} / \mathrm{Kg} \mathrm{PC}$, dix minutes avant l'injection de l'acide acétique par voie intrapéritonéale.

$\checkmark$ Lot 5: souris traitées avec de la naloxone et CAJ à 150 mg/Kg PC, dix minutes avant l'injection de l'acide acétique par voie intrapéritonéale.

\section{Test de l'activité anti-inflammatoire}

L'étude de l'activité anti-inflammatoire de CAJ a été menée suivant la méthode décrite par Lee et al. (2018). Les rats ont reçu l'extrait aqueux des tourteaux de CAJ aux doses allant de 100 à $300 \mathrm{mg} / \mathrm{kg}$ par voie orale ou du diclofénac à une concentration de $75 \mathrm{mg} / \mathrm{kg}$ PC par voie intrapéritonéale, une heure avant l'injection de $0,05 \mathrm{~mL}$ de carragénine (agent d'induction de l'inflammation aiguë) à $1 \%$ sous l'aponévrose plantaire de la patte postérieure droite du rat. Les volumes de la patte ont été relevés avant puis pendant chaque heure durant 5 heures après l'injection de la carragénine, à l'aide d'un pied à coulisse à affichage électronique de marque INGCO. L'activité anti-inflammatoire a été évaluée en pourcentage de réduction de l'œdème chez des rats traités par rapport aux témoins blancs selon la formule suivante:

Pourcentage $(\%)$ d'inhibition $=\frac{C-C 1}{C} \times 100$ $\mathrm{C}=$ pourcentage $(\%)$ d'augmentation moyenne de la circonférence de la patte œedématiée du groupe témoin (lot 1 en un temps donnée).

$\mathrm{C} 1=$ pourcentage $(\%)$ d'augmentation moyenne de la circonférence de la patte œdématiée du groupe test au même moment.

\section{Test de l'activité antipyrétique}

L'injection sous cutanée d'une suspension de levure de bière entraine une hyperthermie chez la souris. Les substances naturelles antipyrétiques et des molécules de référence dont l'aspirine, réduisent cette hyperthermie.

Réparties en cinq (5) lots de 5 souris par lot, elles reçoivent en injection sous cutanée dans la région dorsolatérale une suspension aqueuse de levure de bière à $20 \%$ à raison de $0,1 \mathrm{ml}$ pour $10 \mathrm{mg}$ PC. Puis, elles sont mises à jeun. L'effet pyrogène de la levure de bière engendre un accroissement de la température chez des souris après dix-huit heures de jeûne. Chaque lot de rats reçoit une dose unique de la substance test $(50,100$ et $200 \mathrm{mg} / \mathrm{Kg}$ PC) ou de 
la molécule de référence, l'aspirine $(75 \mathrm{mg} / \mathrm{Kg}$ $\mathrm{PC})$. Le lot témoin reçoit de l'eau distillée.

Les températures rectales sont relevées 1, 2, 3 et 4 heures après l'administration des substances tests, à l'aide d'un thermomètre électronique de précision. La température initiale avant l'administration de la levure de bière a été également relevée.

\section{Analyses statistiques}

Les données statistiques exprimées en moyennes \pm erreur standard $(\mathrm{M} \pm \mathrm{ESM})$ ont été obtenues à partir des $(n=3)$ expériences séparées. Les courbes et l'analyse statistique ont été réalisées avec le logiciel GraphPad Prism 5.01, San Diego, CA, USA. Les moyennes calculées ont été comparées à partir $\mathrm{du}$ test ( $\mathrm{t}$ ) de Student. Lorsque $\mathrm{p} \leq 0,05$, la différence est dite significative.

\section{RESULTATS}

Toxicité aigüe par injection intrapéritonéale de l'extrait aqueux des tourteaux de la pomme de cajou (CAJ)

L'injection intrapéritonéale de l'extrait aqueux des tourteaux de la pomme de cajou, aux doses allant jusqu'à 1000 mg/Kg PC, n'a entrainé aucun mort de souris. Ces tests de toxicité réalisés, conformément au protocole 423 de l'OCDE (OCDE, 2001) permettent d'affirmer que l'extrait aqueux de CAJ, est non toxique

\section{Activité analgésique de l'extrait aqueux des tourteaux de la pomme de cajou (CAJ)}

Le Tableau 1 montre l'activité analgésique chez des souris traitées avec CAJ ou des antalgiques de références. Le nombre important de contorsions provoquées par l'injection de l'acide acétique en présence de l'eau distillée indique de l'effet algogène de ce produit chimique. L'administration orale de l'aspirine à $75 \mathrm{mg} / \mathrm{Kg} \mathrm{PC}$, dix minutes avant l'injection de l'acide acétique, agent algogène, inhibe significativement de 68,96\% $(\mathrm{P}<0,01)$ les contorsions induites (douleur). La réduction est encore importante avec la présence de tramadol (15 $\mathrm{mg} / \mathrm{Kg} \mathrm{PC})$, un antalgique morphinique, affichant un pourcentage d'inhibition de 93,10\% (P<0,001). L'administration préalable de CAJ pour des concentrations comprises entre 75 et 150 $\mathrm{mg} / \mathrm{Kg} \mathrm{PC}$, diminue de manière dosedépendante les contorsions abdominales chez la souris. Cette inhibition significative varie entre 55,17 et $79,31 \%(\mathrm{P}<0,01)$.

Le Tableau 2 montre l'activité analgésique chez des souris traitées avec CAJ ou des antalgiques de références en présence de la naloxone. Le nombre de contorsions abdominales (28) obtenu en présence de la naloxone est sensiblement proche de celui de l'animal témoin (29). En présence de la naloxone, antagoniste morphinique, l'effet protecteur du tramadol contre la douleur induite par l'acide acétique a été réduit significativement de 48,28\% (P <0,01). Dans les mêmes conditions, l'effet protecteur de l'aspirine, a été réduit de façon moins significative de 6,9\%. De même, en présence de la naloxone, l'effet protecteur de CAJ pour des concentrations allant de 75 à $150 \mathrm{mg} / \mathrm{Kg}$ $\mathrm{PC}$ a été réduit de $6,9 \%$ à $7,07 \%$, valeurs non significatives selon l'analyse statistique.

\section{Activité anti-inflammatoire de l'extrait aqueux tourteaux de la pomme de cajou (CAJ)}

La Figure 1 montre l'activité antiinflammatoire chez le rat traité avec le diclofénac (Diclo) ou l'extrait aqueux des tourteaux de pomme de cajou (CAJ). En présence de l'eau distillée, l'injection sous plantaire de la carragenine à $1 \%$, chez le rat (témoin), provoque un œdème dont le volume augmente progressivement avec le temps. Les volumes de la patte sont de $6,18 \mathrm{~mm}$ et 6,45 $\mathrm{mm}$ respectivement au bout de 2 et 3 heures. Le volume maximal de cette augmentation de $7,81 \mathrm{~mm}$, est enregistré à la cinquième heure. En présence de CAJ à $300 \mathrm{mg} / \mathrm{Kg} \mathrm{PC}$, les volumes de la patte ne sont plus que de 5,32 $\mathrm{mm}$ au bout de 2 heures et $5,30 \mathrm{~mm}$ au bout de 3 heures après traitement. En revanche, pour les mêmes temps, en présence de diclofénac à $75 \mathrm{mg} / \mathrm{Kg}$ PC, les volumes de la patte de 
l'animal sont respectivement de $5,43 \mathrm{~mm}$ et $5,05 \mathrm{~mm}$.

Le Tableau 3 présente les pourcentages de réduction de l'augmentation du volume de la patte en présence de CAJ ou de Diclofénac. Cette réduction semble dose-dépendante pour CAJ, d'autant plus qu'elle est importante en présence d'une forte concentration de CAJ $(300 \mathrm{mg} / \mathrm{Kg} \mathrm{PC})$. Trois heures de temps après l'induction de l'inflammation, les réductions de l'œdème, en présence de CAJ pour des concentrations de 200 et $300 \mathrm{mg} / \mathrm{Kg}$ PC, sont respectivement de $47,35 \%$ et $56,22 \%$. L'effet anti-inflammatoire de CAJ à $300 \mathrm{mg} / \mathrm{Kg} \mathrm{PC}$ rappelle celui de Diclofénac à $75 \mathrm{mg} / \mathrm{Kg} \mathrm{PC}$. Toutefois cet effet anti-inflammatoire matérialisé par une réduction de $56,22 \pm 4,3 \%$ $(\mathrm{P}<0,05)$ de l'œdème à la troisième heure, semble moins significatif que celui du Diclofénac à $75 \mathrm{mg} / \mathrm{Kg}$ PC $(66,36 \pm 3,8)$. En revanche, il est significatif $(81,11 \pm 6,9 \%(\mathrm{P}<$ $0,01))$ que celui de Diclofénac $(57,06 \pm 6,8 \%)$, à la cinquième heure.

\section{Activité antipyrétique de l'extrait aqueux des tourteaux de pomme de cajou (CAJ)}

L'activité antipyrétique chez la souris traitée avec CAJ ou l'aspirine (AS), est représentée à la Figure 2. La levure de bière provoque, après 18 heures de jeûne, l'augmentation de la température rectale de la souris. Chez la souris témoin ayant reçue de l'eau distillée comme traitement, la fièvre induite par la levure de bière se maintien audelà de $38^{\circ} \mathrm{C}$ pendant plus de quatre heures. En présence de $\mathrm{CAJ}$, pour des concentrations comprises entre 50 et $200 \mathrm{mg} / \mathrm{Kg} \mathrm{PC}$, l'hyperthermie diminue pour se maintenir autour de $37{ }^{\circ} \mathrm{C}$, à la quatrième heure après traitement. Ces mêmes effets sont observés avec l'aspirine (AS), la molécule de référence.

Le Tableau 4 indique la réduction de la pyrexie chez la souris traitée avec l'extrait aqueux des tourteaux de pomme de cajou (CAJ) ou l'aspirine (AS). CAJ à $50 \mathrm{mg} / \mathrm{Kg} \mathrm{PC}$ présente un effet antipyrétique faible, comparativement à celui de l'aspirine (75 $\mathrm{mg} / \mathrm{Kg} \mathrm{PC}$ ). Le pourcentage faible de réduction de l'hyperthermie en présence de de CAJ à 50 $\mathrm{mg} / \mathrm{Kg} \mathrm{PC}$ est en effet de $34,91 \%(\mathrm{P}<0,01)$ contre $77,85 \pm 4,1 \%$ pour l'aspirine à $75 \mathrm{mg} / \mathrm{Kg}$ $\mathrm{PC}$, après une heure de traitement. En revanche, l'effet antipyrétique de $\mathrm{CAJ}$ à 200 $\mathrm{mg} / \mathrm{Kg}$ PC, est important, mais il est moins significatif que celui de l'AS (75 mg/Kg PC), selon l'analyse statistique. Le pourcentage de réduction de l'hyperthermie par l'AS de $62,95 \pm 6,7 \%$ contre $55,04 \pm 7,4 \%(\mathrm{P}<0,05)$ de $\mathrm{CAJ}$, après cinq heures de traitement, semble le témoigner.

Tableau 1: Activité analgésique chez des souris traitées avec l'extrait aqueux des tourteaux de la pomme de cajou $(\mathrm{CAJ})$ et des antalgiques de références.

\begin{tabular}{lcc}
\hline Traitements & Nombre de contorsion & Pourcentage d'inhibition (\%) \\
\hline Lot 1: Eau distillée & 29 & - \\
Lot 2: Aspirine $75 \mathrm{mg} / \mathrm{Kg} \mathrm{PC}$ & 9 & $68,96^{* *}$ \\
LOt3 : Tramadol 15 mg/Kg PC & 2 & $93,10^{* * *}$ \\
Lot 4: CAJ 75 mg/Kg PC & 13 & $55,17^{* *}$ \\
Lot 5: CAJ $150 \mathrm{mg} / \mathrm{Kg}$ PC & 6 & $79,31^{* *}$
\end{tabular}

Comme l'aspirine et le tramadol, CAJ, diminue significativement le nombre de contorsion induite par l'injection de l'acide acétique, chez la souris. CAJ possède un effet protecteur, analgésique au même titre que les molécules de références, aspirine et tramadol. $* \mathrm{P}<0.05 ; * * \mathrm{P}<$ $0.01 ; * * * \mathrm{P}<0.001$. 
Tableau 2: Activité analgésique chez des souris traitées avec l'extrait aqueux des tourteaux de la pomme de cajou (CAJ) et des antalgiques de références en présence de la naloxone.

\begin{tabular}{lccc}
\hline Traitements & $\begin{array}{c}\text { Nombre de } \\
\text { contorsion }\end{array}$ & $\begin{array}{c}\text { Inhibition des } \\
\text { contorsions (\%) }\end{array}$ & $\begin{array}{c}\text { Réduction des } \\
\text { pouvoirs } \\
\text { protecteurs }\end{array}$ \\
\hline Lot 1: Naloxone + Eau distillée & 28 & 3,44 & - \\
Lot 2: Naloxone + aspirine 75 mg/Kg PC & 11 & 62,06 & 6,9 \\
Lot 3: Naloxone + Tramadol 15 mg/Kg PC & 16 & 44,82 & $48,28 * *$ \\
Lot 4: Naloxone + CAJ 75 mg/Kg PC & 15 & 48,27 & 6,9 \\
Lot 5: Naloxone + CAJ 150 mg/Kg PC & 8 & 72,24 & 7,07 \\
\hline
\end{tabular}

En présence du naloxone, le pouvoir protecteur du tramadol contre la douleur induite par l'acide acétique a été réduit significativement de $48,28 \%$. En revanche, en présence de la naloxone, les pouvoirs protecteurs de CAJ et de l'aspirine contre la douleur induite par l'acide acétique, diminuent respectivement de $6,9 \%$ et 7,07\%, valeurs non significatives. CAJ possède un effet analgésique périphérique, à l'image de l'aspirine. $* \mathrm{P}<0.05 ; * * \mathrm{P}<0.01 ; * * * \mathrm{P}<0.001$.
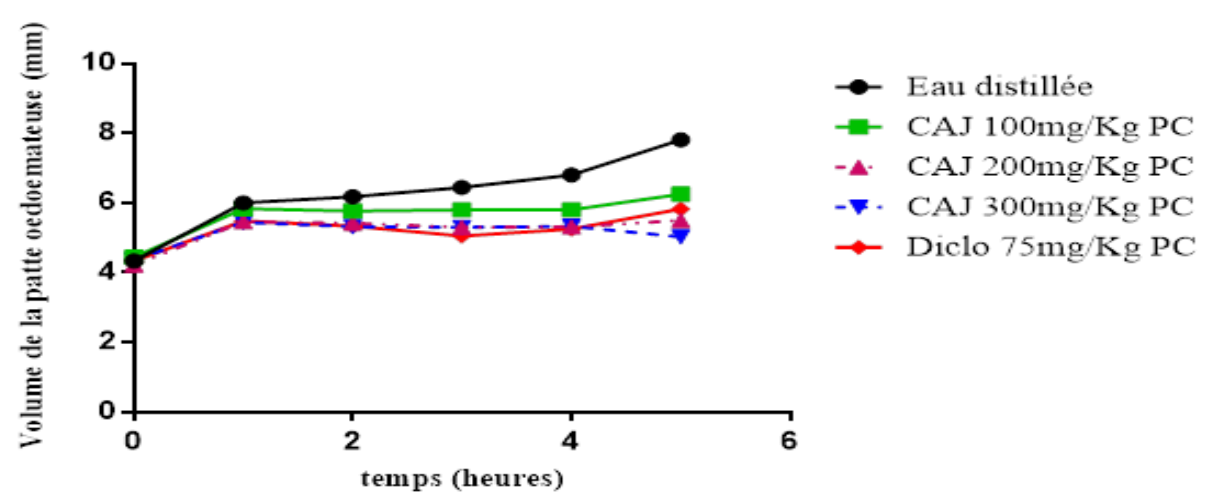

Figure 1 : Activité anti-inflammatoire de l'extrait aqueux des tourteaux de la pomme de cajou (CAJ).

Tableau 3 : Réduction de l'œdème de la patte chez le rat traité avec l'extrait aqueux des tourteaux de pomme de cajou (CAJ) ou le diclofénac (Diclo).

\begin{tabular}{lccccc}
\hline Traitements & \multicolumn{6}{c}{ Réduction de la circonférence de la patte (\%), après traitement } \\
\cline { 2 - 6 } (mg/Kg. P.C.) & $\mathbf{1 h}$ & $\mathbf{2 h}$ & $\mathbf{3 h}$ & $\mathbf{4 h}$ & $\mathbf{5 h}$ \\
\hline CAJ 100 & $18,33 \pm 2,4$ & $30,03 \pm 3,4$ & $37,60 \pm 5,6^{* *}$ & $46,48 \pm 5,4^{*}$ & $49,41 \pm 3,3$ \\
CAJ 200 & $23,16 \pm 3,6$ & $32,93 \pm 4,2^{*}$ & $47,35 \pm 6,4^{*}$ & $54,44 \pm 6,1$ & $62,06 \pm 3,2$ \\
CAJ 300 & $35,38 \pm 2,7$ & $49,58 \pm 5,6$ & $56,22 \pm 4,3^{*}$ & $61,25 \pm 4,9$ & $81,11 \pm 6,99 * *$ \\
Diclo 75 & $30,72 \pm 5,4$ & $45,64 \pm 3,7$ & $66,36 \pm 3,8$ & $63,02 \pm 5,8$ & $57,06 \pm 6,8$ \\
\hline
\end{tabular}

L'effet antiinflammatoire de CAJ à $300 \mathrm{mg} / \mathrm{Kg}$ PC rappelant celui de Diclo à $75 \mathrm{mg} / \mathrm{Kg} \mathrm{PC}$, montre que cet effet est significatif à des concentrations élevées. Chaque valeur représente la moyenne \pm SEM (erreur standard sur la moyenne), $\mathrm{n}=3$. $* \mathrm{P}<0.05 ; * * \mathrm{P}<0.01 ; * * * \mathrm{P}<$ 0.001 . 


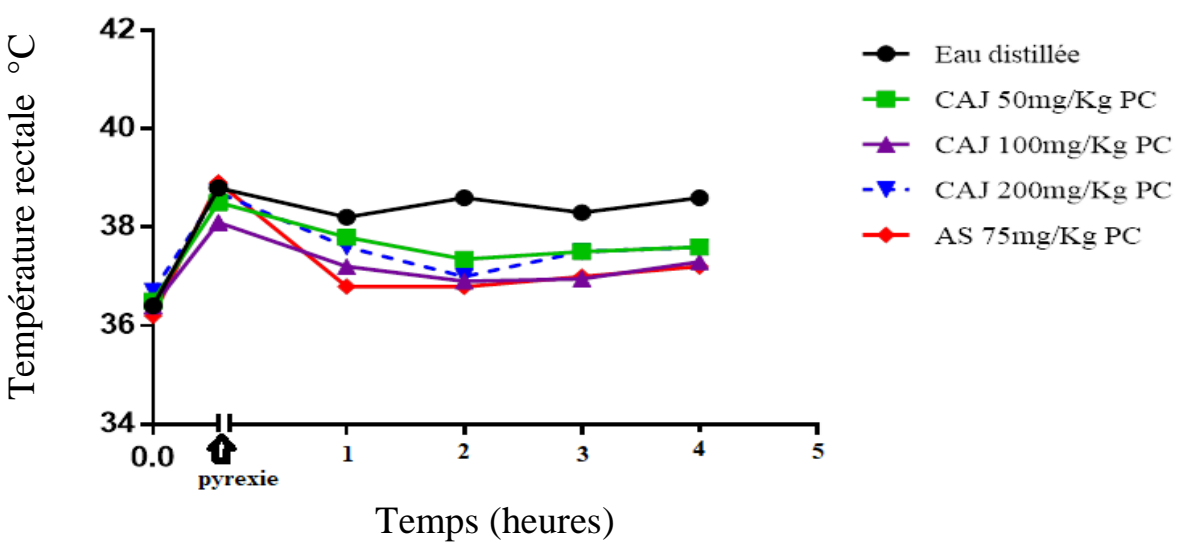

Figure 2 : Activité antipyrétique de l'extrait aqueux des tourteaux de la pomme de cajou (CAJ).

Tableau 4 : réduction de la pyrexie chez la souris traitée avec l'extrait aqueux des tourteaux de pomme de cajou (CAJ) ou l'aspirine (AS).

\begin{tabular}{lcccc}
\hline Traitement & \multicolumn{4}{c}{ Réduction de la température (\%), après traitement } \\
\cline { 2 - 5 } (mg/Kg P.C.) & $\mathbf{1 h}$ & $\mathbf{2 h}$ & $\mathbf{3 h}$ & $\mathbf{4 h}$ \\
\hline CAJ : 50 & $34,91 \pm 3,4^{* *}$ & $37,47 \pm 4,1^{* *}$ & $49,90 \pm 2,4^{* *}$ & $44,97 \pm 5,5^{* *}$ \\
CAJ : 100 & $55,10 \pm 2,8^{* *}$ & $70,66 \pm 5,3$ & $67,66 \pm 4,4$ & $47,10 \pm 4,4^{*}$ \\
CAJ : 200 & $55,04 \pm 1,6^{*}$ & $85,13 \pm 3,7$ & $60,18 \pm 6,3$ & $55,04 \pm 7,4^{*}$ \\
AS : 75 & $77,85 \pm 4,1$ & $78,25 \pm 6,4$ & $70,46 \pm 3,2$ & $62,95 \pm 6,7$
\end{tabular}

$\mathrm{AS}$ et $\mathrm{CAJ}$ possèdent des effets antipyrétiques. Toutefois, l'effet antipyrétique de CAJ est moins significatif que celui de l'AS à la $1^{\text {er }} \mathrm{h}$ et $4^{\mathrm{ème}} \mathrm{h}$. AS est donc plus efficace que CAJ. Chaque valeur représente la moyenne $\pm \mathrm{SEM}$ (erreur standard sur la moyenne), $\mathrm{n}$ $=3, * \mathrm{P}<0.05 ; * * \mathrm{P}<0.01 ; * * * \mathrm{P}<0.001$.

\section{DISCUSSION}

L'objectif de cette étude était d'évaluer les activités analgésique, anti-inflammatoire et antipyrétique de l'extrait aqueux de CAJ.

L'injection de l'acide acétique provoquant des mouvements de contorsions chez la souris atteste de l'effet algogène de ce produit chimique. Des études précédentes notamment celles de Ouédraogo et al. (2012) et Soro et al. (2009), ont donné des résultats similaires. Il est donc indiqué pour évaluer l'activité analgésique de CAJ. En présence de l'extrait aqueux des tourteaux de la pomme de cajou aux concentrations comprises entre 75 et $150 \mathrm{mg} / \mathrm{Kg} \mathrm{PC}$, les crampes abdominales induites par l'acide acétique chez la souris, diminuent de manière dose-dépendante. Ces mêmes effets antagonistes de la douleur, sont également enregistrés en présence de l'aspirine à $75 \mathrm{mg} / \mathrm{Kg} \mathrm{PC}$ et le tramadol à $15 \mathrm{mg} / \mathrm{kg}$ PC . L'extrait aqueux des tourteaux de la pomme de cajou, au vu de ces données, présente des effets analgésiques, au même titre que ces molécules de références, aspirine et tramadol.

Pour caractériser cet effet analgésique de l'extrait aqueux des tourteaux de la pomme de cajou, le test du writhing a été repris en présence de la naloxone, un antagoniste morphinique. Cette molécule est sans effet propre, d'autant plus que les résultats du test de writhing réalisé uniquement en sa présence, rappellent ceux de l'animal témoin, n'ayant reçu que de l'eau distillée comme traitement. Le tramadol dérivé opiacé, en présence de cet 
antagoniste morphinique, affiche un effet analgésique réduit de moitié. En revanche, dans les mêmes conditions, l'extrait aqueux des tourteaux de la pomme de cajou et l'aspirine n'ont pas perdu leur pouvoir protecteur contre les douleurs induites par l'acide acétique. Ces résultats suggèrent que l'effet analgésique de cette substance naturelle est de type périphérique. De ce fait, l'extrait aqueux des tourteaux de la pomme de cajou à l'image des salicylés avec pour chef de file l'aspirine, inhiberait les cyclo-oxygénases inductives et ou constitutives (Franzotti et al., 2002 ; Gupta et al., 2005). Cette inhibition impacterait la libération subséquente des prostaglandines dans le milieu, comme le font remarquer Soro et al. (2009). Les prostaglandines semblent en effet produire une vasodilatation, augmenter la perméabilité vasculaire et stimuler la douleur induite par les autres médiateurs de l'inflammation. Des données semblables ont été obtenues par Parimalakrishnan et al. (2007) travaillant sur Cleome chelidonii.

Les tests anti-inflammatoires indiquent que $\mathrm{CAJ}$ et le diclofénac, réduisent l'œdème induit par la carragénine. La réduction de l'œdème pourrait s'expliquer, par l'inhibition des médiateurs de l'inflammation notamment l'histamine issue de la de granulation des basophiles et des mastocytes locaux, les sérotonines produites par désintégration des plaquettes sanguines, durant les premières heures de l'inflammation, et de la bradykinine libérée par un système protéique du plasma, au cours des heures intermédiaires. En revanche, l'inflammation serait contenue par l'inhibition de la cyclo-oxygénase inductible, qui libère in fine à partir de l'acide arachidonique, la prostaglandine, durant les dernières heures, (Wantana et al., 2009). CAJ affichant une plus forte réduction de l'œdème durant ces dernières heures, possèderait une action antiinflammatoire prépondérante, liée à l'inhibition de cet enzyme, tout comme les AINS y compris le diclofénac (Okombe et al., 2019). L'effet antiinflammatoire de CAJ à 300 $\mathrm{mg} / \mathrm{Kg}$ PC rappelant celui de Diclofénac à 75 $\mathrm{mg} / \mathrm{Kg} \mathrm{PC}$, montre qu'il est significatif à des concentrations relativement élevées, à l'image de Ximenia Américana (Soro et al., 2015). Avec une réduction de l'œdème de la patte de rat de 56,22\%, à la troisième heure de l'expérience, on en déduit que CAJ pourrait également contenir des composés antibradykinines. Concernant $\mathrm{Ce}$ mode d'action de CAJ, il pourrait être différent de ceux de Ximenia Américana (Soro et al., 2015) et de Vitex congolensis De Wild (Okombe et al., 2019) dont les activités anti-inflammatoires se manifestant dès les premières heures, pourraient être dirigées contre la production de la sérotonine et de l'histamine. Phragmanthera capitata (Etame et al., 2018), et Zanthoxylum zanthoxyloides (Diatta et al., 2014) sont également des substances naturelles de la pharmacopée connues pour leurs activités antiinflammatoires.

La fièvre accompagnant les réactions inflammatoires explique l'évaluation de l'effet antipyrétique de CAJ. La levure de bière, agent pyrogène provoque, une élévation de la température corporelle chez des souris, après dix-huit heures de jeûne. Injectée dans la zone dorsolatérale, elle induit la formation de pyrogènes endogènes dont l'interleukine 1 , la TNFa qui suscitent la production de prostaglandines cérébrales, d'où l'augmentation de la température (Ribeiro et al., 2010). Comme les salicylés, l'extrait aqueux de CAJ, diminuant la température rectale des souris, pourrait interférer sur ce mécanisme en inhibant la production de la prostaglandine. Toutefois, au regard des données de l'analyse statistique, l'effet antipyrétique de CAJ est moins efficace que celui de l'aspirine. Selon Ouédraogo et al. (2012) Pterocarpus erinaceus Poir. (Fabaceae) aurait tout aussi un effet antipyrétique.

L'effet antalgique de CAJ, pourrait être lié en sa richesse en saponosides et flavonoïdes (Dosso et al., 2021). En outre, il serait non toxique au vu des données toxicologiques.

\section{Conclusion}

L'extrait aqueux des tourteaux de la pomme de cajou, par injection intrapéritonéale 
est non toxique. Il possède des propriétés analgésique, antipyrétique et antiinflammatoire liées probablement à son contenu phytochimique, indiquant la présence de saponosides et de flavonoïdes. Ces données pharmacologiques de CAJ, montrent la place que pourrait occuper les agro-ressources dans la consolidation de notre santé, en particulier dans la prise en charge de la douleur, de la fièvre et de l'inflammation.

\section{CONFLIT D'INTERETS}

Les auteurs déclarent qu'ils n'ont aucun conflit d'intérêts.

\section{CONTRIBUTIONS DES AUTEURS}

Les auteurs MD, AEK et AT ont conduit les expérimentations, contribuées à la récolte des données ainsi qu'à la rédaction du manuscrit. Quant aux auteurs DS et ND, ils ont aidé à l'analyse statistique des données et à la correction du manuscrit. La version finale du manuscrit a été approuvée par tous les auteurs.

\section{REMERCIEMENTS}

Les auteurs remercient $\mathrm{Mr}$ Odroko Gnoezele Freddy, technicien de l'animalerie de l'UFR des Sciences Pharmaceutiques et Biologiques de 1'Université Félix Houphouët Boigny (SPB) pour sa contribution dans la réalisation des différents tests (antiinflammatoire, antipyrétique et analgésique).

\section{REFERENCES}

Chira K, Suh JH, Saucier C, Teissèdre PL. 2008. Les polyphénols du raisin. Phytothérapie, 6: 75-82. DOI: 10.1007/S10298-008-0293-3

Corrado B, Marco T, Colucci R, Fornai M, Antonioli L, Ghisu N, Del Tacca M. 2009. Role of coxibs in the strategies for gastrointestinal protection in patients requiring chronic nonsteroidal anti-infllammatory therapy. Pharmacol. Res., 59: 90-100. DOI : $10.1016 /$ j.phrs.2008.11.004
Diatta W, Sy GY, Manga CI, Diatta K, Dior A, Bassene E. 2014. Recherche des activités anti-inflammatoire et analgésique des extraits de feuilles de Zanthoxylum zanthoxyloides (Lam) zepernick et timler (Rutaceae). Int. J. Biol. Chem. Sci., 8(1): 128-133. DOI: 10.4314/ijbcs.v8i1.12

Dosso M, Niamke AM, Soro D, Ouattara PGH, Coulibaly A. 2021. Autoregulatory glycemic activity of the aqueous extract of cashew apple cake (Anacardium occidentale L.). JPP., 10(3): 56-62.

Etame LG, Ngaba GP, Kamdom M, Mpondo ME, Dibong SD. 2018. Evaluation des activités antiinflammatoire et antiradicalaire de l'extrait au vin de palme des feuilles de Phragmanthera capitata (Sprengel) S. Balle (Loranthaceae) récoltées sur $P$. guajava au Cameroun. Int. J. Biol. Chem. Sci., 12(1): 233-243. DOI: https://dx.doi.org/10.4314/ijbcs.v12i1 .18

Franzotti EM, Santos CVF, Rodrigues HMSL, Mourao RHV, Andrade MR, Antoniolli AR. 2002. Antiinflammatory, analgesic and acute toxicity of Sida cardiafolia L. J. Ethnopharmacol., 72: 273-278.

Gupta M, Mazumder UK, Sambath KR, Gomathi P, Rajeshwar Y, Kakoti BB, Selven VT. 2005. Anti-inflammatory, analgesic and antipyretic effects of methanol extract from Bauhinia racemosa stem bark in animal models. J. Ethnopharmacol., 98: 267-273. DOI : 10.1016/j.jep.2005.01.018

Lee YY, Saba E, Irfan M, Kim M, Chan JY, Jeon BS, Choi SK. 2018. The antiinflammatory and anti-nociceptive effects of Korean black ginseng. Phymed., 54: 169-181. https://www.ncbi.nlm.nih.gov/pubme $\mathrm{d} / 30668366$ 
OCDE. 2001. Ligne directrice de l'OCDE pour les essais de produits chimiques, Norme 423. OCDE, Paris-France.

Okombe EV, Nzuzi MG. 2019. Evaluation de l'activité antipyrétique, analgésique et anti-inflammatoire de l'extrait méthanolique de Vitex congolensis De Wild. \& T. Durand. Int. J. Biol. Chem. Sci., 13(7): 3066-3078. DOI: https://dx.doi.org/10.4314/ijbcs.v13i7 .8

Ouédraogo N, Lompo M, Sawadogo RW, Tibiri A, Hay AE, Koudou J, Dijoux MG, Guissou IP. 2012. Etude des activités anti-inflammatoire, analgésique et antipyrétique des décoctés aqueux des feuilles et des racines de Pterocarpus erinaceus Poir. (Fabaceae). Phytothérapie, 10: 286292. DOI :10.1007/S10298-0120732-Z

Parimalakrishnan S, Akalanka D, Anton SA, Manavalan R. 2007. Evaluation of anti-inflammatory, antinociceptive and antipyretic effects of methanol extract of Cleome chelidonii. Int. J. Biol. Chem. Sci., 1(3): 223-228.

Ramalawi M, Marti C, Sarasin F. 2013. Intoxication aiguë au paracétamol. Rev. Med. Suisse, 9: 1478-82.

Ribeiro RV, Matos da Silva R, Corsino da Silva JL, Tabajara de Oliveira MD. 2010.

Antiinflammatory, antinociceptive and antipyretic effects of hydroethanolic extract from Macrosiphonia velame (A. St. Hil.) M. Arg. in animal models. Brazil. $J$ Pharmaceut. Sci., 46: 515-23. DOI : https://doi.org/10.1590/S198482502010000300015

Soro D. 2012. Couplage de procédés membranaires pour la clarification et la concentration du jus de pomme de cajou, Performances et impacts sur la qualité des produits. Thèse de Doctorat, Université Montpellier Supagro, France, 156 p.

Soro TY, Traore F, Sakande J. 2009. Activité analgésique de l'extrait aqueux de Ximenia americana (Linné) (Olacaceae). C. R. Biologies, 332: 371-377. DOI: 10.1016/j.crvi.2008.08.022

Soro TY, Néné-bi AS, Zahoui OS, Yapi A, Traoré F. 2015. Activité antiinflammatoire de l'extrait aqueux de Ximenia americana (Linné) (Olacaceae). Journal of Animal \& Plant Sciences, 24(3): 3802-3813.

Soro TY, Traoré F, Datte JY, Nene-Bi AS. 2009. Activité antipyrétique de l'extrait aqueux de Ximenia americana. Phytothérapie, 7: 297303. DOI: 10.1007/s10298-009-05073

Tédong L, Djomeni DDP, Dimo T, Asongalem AE, Sokeng SN, Flejou JF, Callard P, Kamtchouing P. 2007. Effet de l'extrait à l'hexane des feuilles d'Anacardium occidentale L. (Anacardiacées) sur la fonction de reproduction chez les rats rendus diabétiques par la streptozotocine. Phytothérapie, 5: 182-193. DOI 10.1007/s10298-007-0251-5

Wantana R, Tassanee N, Subhadhirasakul S. 2006. Antinoceptive, antipyretic, and anti-inflammatory activities of Putranjiva roxburghii Wall. Leaf extract in experimental animals. $J$. Nat. $\quad$ Med., $\quad$ 63: $\quad 290-296$. DOI: $10.1007 / \mathrm{s} 11418-009-0336-6$

Zaouaoui FS. 2005. La Santé par les Produits de la Nature: Les Fruits. Editions Jugurtha International 3: Tunis. 